\title{
Analysis of Spatial Evolution Characteristics of Floating Population in Lanzhou
}

\author{
Hang ZHAO ${ }^{a}$, Zhi-Bin ZHANG ${ }^{\text {b, }}$, Kai-Jia WANG ${ }^{c}$ and Wei-Min GONG ${ }^{d}$ \\ College of Geography and Environmental Science, \\ Northwest Normal University, Lanzhou, China \\ azhaohanghjacket@163.com, ${ }^{\mathrm{b}}$ zbzhang@nwnu.edu.cn, \\ c18393810275@163.com, ${ }^{\mathrm{d}}$ 13993182790@163.com
}

Keywords: Floating population, Spatial evolution, Lanzhou.

\begin{abstract}
This paper analyzed the spatial evolution characteristics of floating population in Lanzhou from the aspects of scale, density, and spatial correlation. The results showed that floating population is in a large scale by contrast and in a remarkable speed of increasing, the number of floating population which the gap enlarge further between blocks distributes obviously in municipal districts. From the aspect of density, the pattern of floating population has changed from single center to multicenter and the density variation is significant, in the same time there exists a character of internal heterogeneity of which the central urban area density is sparse. The spatial autocorrelation of floating population is obvious between blocks, the agglomeration phenomenon is gradually weakened, but the overall pattern of the floating population that is sparser in western area than in eastern area is not broken.
\end{abstract}

\section{Introduction}

Floating population usually refers to the population separated from the place of residence, lasting less than one year or six months of the short-term outgoing population[1-3].With the establishment of socialist market economic system in China and the reform of the household registration system, the city has flowed into a large number of floating population in the process of urbanization. A large-scale floating population has had a significant impact on the process of urbanization, social and economic development, the resources and environment in China. Based on census data gradually increase the refinement and practical investigation, Chinese scholars have studied the effect of the floating population, the spatial distribution and migration factors of floating population in big cities, policy management and other aspects of the impact of floating population[4-11].Existing research results are in the majority with multi-provincial macroscopic scale, while the microscopic scale research mainly are concentrated on the first-tier cities, the research to the northwest inland cities is relatively few. Therefore, from the micro scale, this paper will probe into the spatial differentiation pattern and its evolution characteristics of floating population in Lanzhou, in order to provide reference for urban development, resource allocation, floating population policy and service facilities construction.

\section{Research Area, Data and Methods}

\section{Overview of the Research Area}

Lanzhou, the capital of Gansu Province, is the political, economic and cultural center of Gansu Province. Now it has jurisdiction over Chengguan, Anning, Qilihe, Xigu and Honggu five districts, Yongdeng, Yuzhong, Gaolan three counties. Because of its special geographical location and social and economic attraction, Lanzhou has become the most concentrated region of floating population in Gansu province. In this paper, we choose Chengguan, Qilihe, Xigu and Anning four districts, 48 blocks and 1 high-tech development zone as the research areas.

\section{Data}

The population data in this paper are derived from the fifth and sixth population censuses of 
Lanzhou. The spatial data are based on the second National Land Survey of Lanzhou and the urban basic geographic information. The outflow population selection census data "out of the population for more than half a year". And the inflow population of each block was selected from "this county external population, this province other counties(cities) external population, other province external population" three terms. The floating population is obtained by calculating the inflow minus the difference of the outflow.

\section{Metrology}

Global Spatial Autocorrelation is used to reflect the concentration degree of spatial distribution of a certain index and to reveal the distribution of the observed index in the whole region. At present, the commonly used metric is the Moran's I index, which is formulated as follows:

$$
I=\frac{\sum_{i=1}^{n} \sum_{j \neq 1}^{n} W_{i j}\left(X_{i}-\bar{X}\right)\left(X_{j}-\bar{X}\right)}{S^{2} \sum_{i=1}^{n} \sum_{j \neq 1}^{n} W_{i j}}
$$

In the formula, ' $n$ ' represents the number of the whole research area, $W_{\mathrm{ij}}$ is the spatial adjacent weight value, $\bar{X}$ is the average of the attribute value, $X_{i}$ and $X_{j}$ are the attribute value of the research area ' $\mathrm{i}$ ' and ' $\mathrm{j}$ '. The value of Moran's $\mathrm{I}$ is in [-1,1].If $\mathrm{I}>0$, the floating population has a positive correlation in space, and if $\mathrm{I}<0$, the floating population has a negative correlation in space.

Local Spatial Autocorrelation is used to measure the correlation between a spatial element of a local region and its adjacent spatial elements in the whole research area. Use of Getis-Ord Gi* Index to explore spatial heterogeneity among research regions:

$$
G_{i}^{*}=\frac{\sum_{j=1}^{n} W_{i j} X_{j}}{\sum_{j=1}^{n} W_{i}}
$$

In the formula, $\mathrm{W}_{\mathrm{ij}}$ is the space weight, if the value is significantly positive, it shows that the value of the research area is relatively higher than that of the adjacent research area. It indicates that the region is a high-value spatial agglomeration area(hot spot area).Otherwise, it is a low-value spatial agglomeration area(cold point area).

\section{Spatial Evolution Characteristics}

\section{Quantitative Evolution Characteristic}

The spatial differentiation of the floating population shows the characteristics of agglomeration to the central urban area, and the inner spatial heterogeneity occurs in the old urban area and the suburban area. As is shown in Table 1,the proportion of floating population to the total population was $26.7 \%$ in 2000 , the number was 475000.In 2010,the proportion of floating population to the total population was 25.2\%,the number was 585600.From 2000 to 2010,the number of floating population in Lanzhou has been increasing. The rate of rise was $23.3 \%$,the average annual growth rate was $2.12 \%$,but the proportion of floating population to the total population is decreasing.

The number of floating population in 2010 was 293900 in Chengguan District,112400 in Qilihe District,74000 in Anning District and 42900 in Xigu District. Compared with the scale of floating population in 2000,the floating population in Chengguan District increased by 62300 people, the growth rate was $21.2 \%$ and the average annual growth rate was $1.9 \%$.The floating population in the Qilihe District grew by 11400 people, the growth rate was $11.1 \%$,and the average annual growth rate was $1.01 \%$.The floating population in Anning District grew by 41900 people, the growth rate was $130.53 \%$,the average annual growth rate was $11.87 \%$.The floating population in Xigu District decreased by 4500 ,the growth rate was $-9.5 \%$,the average annual growth rate was -0.86\%.Chengguan and Anning District increased significantly, while Xigu District showed negative growth. 
Table 1. The scale of floating population in Lanzhou

\begin{tabular}{|c|c|c|c|c|}
\hline \multirow[b]{2}{*}{ Region } & 2000 & 2010 & \multirow[b]{2}{*}{$\begin{array}{l}\text { Growth } \\
\text { rate(\%) }\end{array}$} & \multirow[b]{2}{*}{$\begin{array}{l}\text { Average annual } \\
\text { growth rate(\%) }\end{array}$} \\
\hline & $\begin{array}{l}\text { Floating } \\
\text { population }\end{array}$ & $\begin{array}{l}\text { Floating } \\
\text { population }\end{array}$ & & \\
\hline Lanzhou & 476000 & 587000 & 23.3 & 2.12 \\
\hline $\begin{array}{l}\text { Chengguan } \\
\text { District }\end{array}$ & 293900 & 356200 & 21.2 & 1.92 \\
\hline Qilihe District & 102900 & 114300 & 11.1 & 1.01 \\
\hline Xigu District & 47400 & 42900 & -9.5 & -0.86 \\
\hline Anning District & 32100 & 74000 & 130.53 & 11.87 \\
\hline
\end{tabular}

\section{Density Evolution Characteristic}

According to the density distribution of floating population, the average density of floating population increased from 1076.21 people $/ \mathrm{km}^{2}$ in 2000 to 1470 people $/ \mathrm{km}^{2}$ in 2010.The number of high-density blocks of floating population decreased, and the number of low-density blocks increased. In 2000,the density of floating population gradually decreased from high density street such as Jiuquanlu block to peripheral block. In 2010,the center of gravity of floating population density shifted and appeared in Yanbei block, Yannan block, Xihu block, Peili block and other high-density floating population blocks. The floating population in high-density blocks has the characteristics of economic center orientation. In 2000, the floating population in Lanzhou concentrated in the commercial centers such as Zhangyelu block. These regions have many employment opportunities to engage in business, which is conducive to engaging in commercial activities. The spatial agglomeration effect of floating population is obvious. With the construction of Lanzhou high-tech industrial development zone after 2000, the characteristics of floating population "hollowing out" are obvious. The high-density floating population block, which is directed by the commercial center such as Zhangyelu block, is transformed into a low-density zone. The floating population that is employed by migrant working, demolition and relocation and other reasons in Yantan and other blocks was densely distributed.

It can be concluded that the spatial pattern of floating population density in 2000 was that the concentration of single center decreased to the gradient of the periphery. In 2010,it was the pattern of multi-center agglomeration extending to periphery. And it appeared hollow internal collapse phenomenon in the economic core region .

\section{Spatial Correlation Characteristic}

In order to further study the spatial correlation of the floating population, by using Global Spatial Autocorrelation, Moran's I value that it showed significant at 5\% level were 0.147 and 0.142 in 2000 and 2010 respectively, and the test statistic $Z$ value were greater than 1.96.It indicated that the floating population in Lanzhou are positively related to characteristics. From 2000 to 2010, Moran's I value of floating population decreased, it means that floating population gather agglomeration weakened, the spatial distribution trended to diffusion.

The Global Spatial Autocorrelation Moran's I index can not reflect the local spatial correlation of floating population in Lanzhou. The Getis-Ord Gi* index for 2000 and 2010 is calculated and divided into five levels. As is shown in Fig.1,the hot spot area was mainly located in the eastern urban area in 2000,the center of the high concentration hot spot block was located in Yantan town, Gongxingdun block and Weiyuanlu block. The hot spot area and sub-hot spot area scope decreased in 2010.The center of high-concentration hot-spot block moves to north such as Yanchanglu block, Yanbei block, Yannan block. The evolution of the low concentration cold spot block was reduced from east to west, the cold spot block was distributed in Xigu district, part of Anning district and Qilihe district in 2000, and the center of cold spot block was located in Shajingyi block, Liu Jiabao block and Cuijiaya block, etc. In 2010,the cold spot showed two district centers: Xiliugou block and Baiyinlu block, the cold spot area and the sub-cold spot area scope obviously reduced. To sum up,the evolution range of floating population hot spot area is reduced generally, concentrated on Yantan and the north bank of the Yellow River in the eastern urban area, and the cold spot area shows a saltatory evolution, which shrinks in the western urban area, and expands suddenly in the 
eastern of Gaolanlu block and other blocks. On the whole, the general trend remains unchanged that the gradient evolution of the floating population from the hot spots to the west and the cold spots in the eastern urban areas, the scope of the transition areas is expanded, and the ranges of the hot spots and the cold spots are gradually reduced.
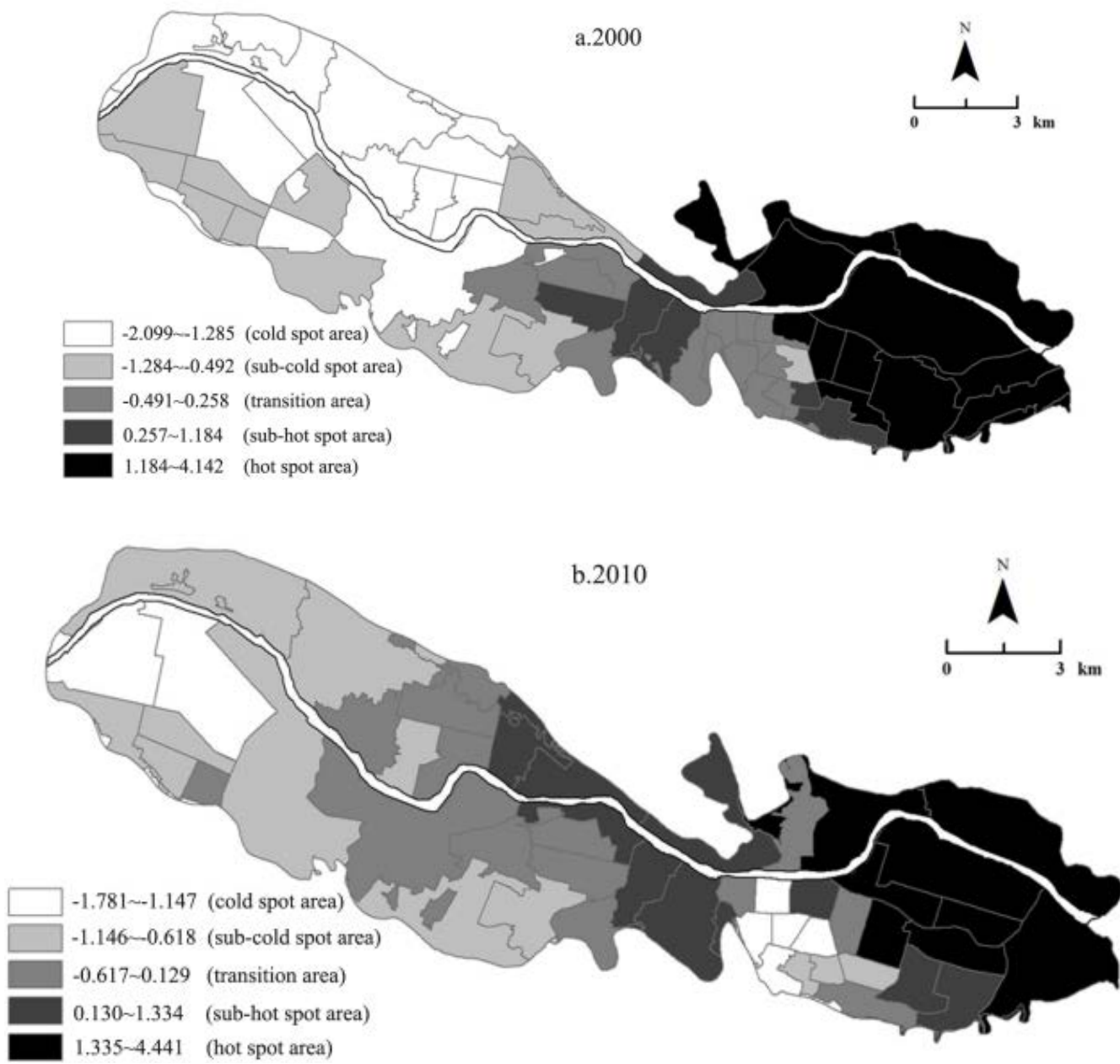

Fig.1 Spatial correlation pattern of floating population in Lanzhou

\section{Summary}

Based on the above analysis methods, the following conclusions are drawn: the scale of floating population in Lanzhou is expanding, the gap of each block floating population tends to ease. The floating population density shows a growth tendency, the number of high density blocks have reduced while the low density blocks have increased. It appears the change of density pattern of floating population from single center agglomeration to multicenter agglomeration. From the view of spatial correlation characteristic, the spatial positive correlation existed in the whole city area, but the spatial agglomeration was weakened.

The city as an influx of settlements, there are differences in the process of social integration among floating population. It is necessary to strengthen urban infrastructure and improve social security systems. The research on the driving mechanism of spatial differentiation of floating population and the qualitative study of floating population under spatial micro-location needs to be further deepened in the future.

\section{Acknowledgment}

This research was financially supported by the National Science Foundation of China(41161028). 


\section{References}

[1] Shan-yu Zhang, Introduction of Population Geography,Shanghai,1999.(In Chinese)

[2] Raven stein E.G, The Laws of Migration[J].Journal of the Royal Statistical Society of London.1885, (6):167-235.

[3] Bogue D. J. The Study of Population: An Inventory and Appraisal[C]. Chicago: University of Chicago Press, 1955:486-509.

[4] Juan Li, Guo-zhu Ren, Qi Lu and Jun-yan Zhang, The research of characteristics and causes of the migrant population in Chongqing[J].Process in geography,2006,(25):77-83.(In Chinese)

[5] Bo Zhao, Lie Chen, Hua-song Luo and You-de Wu, Study on spatial structure of floating population in Kunming[J].World region studies,2004,(13): 66-73.(In Chinese)

[6] Lei Fang, Ke-wei Liu, Ling Xu, Jian-wei Li, The spatiotemporal characteristics and influencing factors of floating population in Shaanxi province-A study on fourth, fifth and sixth census[J].Journal of Shaanxi normal university(natural science edition),2015,(43):92-98.(In Chinese)

[7] Hua-song Yao, Xue-qiang $\mathrm{Xu}$, De-sheng Xue, On spatial distribution and evolutive laws of floating population in Guangzhou[J].Economic geography,2010,(30): 40-45.(In Chinese)

[8] Chuan-geng Zhu, Rong-hua Ma, Feng Zhen, Chao-lin Gu, The spatial structure of the floating population in Chinese cities[J].Human geography,2001,17(1): 65-68.(In Chinese)

[9] Shan-ping Yan, Inteprpvincal migration and its determinants in 1990's China[J].Chinese journal of population science,2007,(1): 71-77.(In Chinese)

[10] Jie-zhu, Spatial structure, mode and the meaning of population migration in the Yangtze river delta[J].Process in geography,2009,28(3): 353-361.(In Chinese)

[11] Bao-yu Xiao, Yu Zhu, Patterns of spatial differentiation of the urban floating population in Fujian province: an analysis of the sixth population census data[J].Human geography,2014,(4): 85-91.(In Chinese) 